\title{
El problema del objetivismo en la producción de conocimiento sociológico: La correspondencia entre Alfred Schutz, Talcott Parsons y Harold Garfinkel
}

\author{
The Problem of Objectivism in the Production of Sociological Knowledge: The \\ correspondence of Alfred Schutz, Talcott Parsons and Harold Garfinkel
}

Dra. Daniela G. López (danielalopez@daad-alumni.de) Instituto de Investigaciones Gino Germani, Universidad de Buenos Aires (Buenos Aires, Argentina)

\begin{abstract}
The epistemological problem of objectivism in the production of sociological knowledge confronts the researcher with the question of the risk involved in substituting social reality by the idealizations and abstractions created by science. Without a doubt, the subject seems intriguing and requires its thematization facing toward and appropriate foundation of sociological concepts. In order to address that problem, the article aims to recover, from a hermeneutic perspective, a phenomenologically inspired epistemology in the works of Alfred Schutz and Harold Garfinkel. To achieve this goal we first analyse Schutzian contributions in the context of the correspondence held with Talcott Parsons. There the author thoroughly discusses the difficulties that social sciences go through regarding the foundation of its concepts. Secondly, we revise unpublished bibliographical heritage from Garfinkel's work who interested in the epistemological debate between Schutz and Parsons- picks up the epistemological problem again. We will sustain that unlike Schutz who proposes a philosophical response consisting in the articulation of an ontology of the life-world, Garfinkel develops a methodological answer which aims to illuminate the practical reasoning and the methodological decisions of researchers in order to avoid the substitution of social reality by the idealities of the scientific reflection.
\end{abstract}

Keywords: Alfred Schutz, Talcott Parsons, Harold Garfinkel, epistemology, phenomenology

\section{Resumen}

El problema epistemológico del objetivismo en la producción de conocimiento sociológico confronta al investigador con la pregunta acerca del riesgo que conlleva la sustitución de la realidad social por las idealidades y las abstracciones creadas por la ciencia. A fin de abordar ese problema, el artículo se propone recuperar, desde una perspectiva hermenéutica, una epistemología de inspiración fenomenológica en los textos de Alfred Schutz y de Harold Garfinkel. Para ello se analizan, en primer 
lugar, los aportes de Schutz en la correspondencia mantenida con Talcott Parsons pues es allí donde el autor discute ampliamente las dificultades por las que atraviesan las ciencias sociales con relación a la fundamentación de sus conceptos. En segundo lugar, se revisan acervos bibliográficos inéditos de la obra de Garfinkel quien, interesado por el debate epistemológico entre Schutz y Parsons, retoma ese mismo problema epistemológico. Sostendremos que, a diferencia de Schutz quien propone una salida filosófica que consiste en la articulación de una ontología del mundo de la vida, Garfinkel elabora una respuesta de carácter metodológico que apunta a iluminar los razonamientos prácticos y las decisiones metodológicas de los investigadores a fin de evitar la sustitución de la realidad social por las idealidades de la reflexión científica.

Palabras clave: Alfred Schutz, Talcott Parsons, Harold Garfinkel, epistemología, fenomenología

\section{Introducción}

El problema epistemológico del objetivismo en la producción de conocimiento sociológico confronta al investigador con la pregunta acerca del riesgo que conlleva la sustitución de la realidad social por las idealidades y las abstracciones creadas por la ciencia. Indudablemente, el tópico resulta sugerente y requiere ser tematizado de cara a una adecuada fundamentación de los conceptos sociológicos.

Convocados por ese problema, en los años 40 y 50, tres importantes referentes de la teoría sociológica contemporánea, Alfred Schutz, Talcott Parsons y Harold Garfinkel, intercambiaron reflexiones críticas acerca de sus principales aristas. Pocas veces se ha tenido la posibilidad de leer los intercambios de opiniones de autores tan destacados en el ámbito de la reflexión sociológica discutiendo acerca de tópicos epistemológicos de tanta centralidad para la fundamentación del conocimiento científico.

A fin de analizar el modo en el que cada uno de estos autores da respuesta a esos problemas, el trabajo se propone, en la primera parte, revisar el estudio crítico de Schutz a Parsons señalando como principal eje el problema del objetivismo en la producción de conocimiento. En ese manuscrito Schutz plantea la importancia de clarificar el sentido subjetivo, de modo de evitar el "olvido" y la sustitución de la realidad social. Clarificación que, a juicio del autor, solo puede llevarse a cabo a partir de la comprensión filosófica. En ese marco se resalta el modo en el que las cartas muestran el interés de Schutz de fundamentar filosóficamente, o en sus propias palabras, "ensanchar", "profundizar" y "complementar" el marco de referencia parsoniano de modo de recuperar ese fundamento de sentido "olvidado".

Asimismo, se señala que, a pesar de esa intención de complementariedad, los intérpretes y comentaristas de la correspondencia ofrecieron una comprensión distorsionada de la misma y la analizaron desde una perspectiva dualista. En ese sentido, se muestra el modo en que la teoría social ha sostenido una interpretación dominante de la fenomenología a través de lo que puede denominarse como "lecturas comunitarias" erróneas de sus textos centrales, las que refieren a un "consenso virtual" basado en profundos malentendidos que han sido sostenidos a través de un conjunto común de eslóganes. 
López, D. 2014. El problema del objetivismo en la producción de conocimiento sociológico: La correspondencia entre Alfred Schutz, Talcott Parsons y Harold Garfinkel

En contraposición a este consenso extendido, el artículo enfatiza que el propósito de trazar un puente entre las categorías subjetivas y objetivas de modo de superar el problema del objetivismo en la producción de conocimiento en ciencias sociales fue un objetivo que Schutz se planteó de modo muy claro ya en su obra temprana. Para ello se presenta un examen de las discusiones que motivaron su obra de juventud las que permiten demostrar que el problema del objetivismo y la superación del dualismo fue el tópico central que movilizó su proyecto teórico y epistemológico.

En la segunda parte, el artículo revisa acervos bibliográficos inéditos: los intercambios que Schutz mantuvo con Garfinkel. En ellos no solo es posible encontrar una interpretación retrospectiva de Schutz sobre su correspondencia con Parsons a la luz del problema del objetivismo en la producción de conocimiento en ciencias sociales, sino que también se revela que esa problemática ocupaba un lugar central en la reflexión de quien más tarde sería el fundador de la etnometodología. Los intercambios entre Schutz y Garfinkel respecto de la correspondencia entre Schutz y Parsons permiten, en primer lugar, apoyar la insistencia de Schutz en torno a su afinidad y complementariedad con el pensamiento de quien, desde la interpretación dominante, es su oponente. Por otra parte, presentan el enorme potencial de mostrar "en germen" el análisis garfinkeliano al problema del objetivismo en la producción de conocimiento. El artículo recupera y revisita dichos acervos bibliográficos inéditos de la obra temprana de Garfinkel, los cuales han sido escasamente estudiados en el ámbito de la discusión especializada, a fin de rastrear en ellos una epistemología de inspiración fenomenológica que ofrezca un abordaje empírico distintivo para investigar la producción de conocimiento en sociología.

\section{La correspondencia Schutz-Parsons}

La correspondencia entre Schutz y Parsons puede ubicarse en el contexto del advenimiento de la Segunda Guerra Mundial, las secuelas de la gran depresión y el éxodo de los científicos sociales europeos en Estados Unidos. En ese tiempo, Schutz era un intelectual vienés inmigrante que se instalaba con su familia en Nueva York y Parsons un científico social que gozaba de una posición privilegiada en la Universidad de Harvard. En el año 1938, durante su exilio en París, Schutz había leído La Estructura de la Acción Social (en adelante EAS) publicado en el año 1937. Fue a causa de esta lectura que Schutz vio en Parsons a un pensador con intereses afines, un autor con raíces weberianas que había demostrado la importancia del abordaje "subjetivo". Además, algo que resultaba alentador para Schutz era el hecho de que Parsons había listado su primer libro La Construcción Significativa del Mundo Social (en adelante CSM) publicado en 1932 en la bibliografía de su estudio de 1937.

El primer encuentro entre ambos debió haber ocurrido en septiembre de 1939 durante un congreso de sociología en Boston. Sin embargo, el estallido de la guerra obligó a Schutz a regresar a New York antes de que tal encuentro pudiera concretarse. En los meses subsiguientes comenzó la correspondencia. En una carta de septiembre de 1939, Schutz le manifiesta a Parsons el interés en su trabajo y lo pone al corriente de la escritura de un estudio crítico a EAS, estudio que desencadenará la correspondencia entre ambos autores. La redacción del estudio crítico había comenzado con una invitación a fines del año 1938 del Profesor Friedrich Von Hayek, entonces editor de la revista Economica de la London School of 
López, D. 2014. El problema del objetivismo en la producción de conocimiento sociológico:

Economics, quien le propone a Schutz escribir una reseña del libro recientemente publicado por Parsons. El trabajo, según explica Schutz, debía tener una extensión máxima de 4.000 palabras. Sin embargo, el resultado fue un artículo de 20.000 palabras.

El estudio crítico a EAS dio lugar a un intercambio que duró solo unos pocos meses, desde el 15 de noviembre de 1940 hasta el 21 de abril de 1941. No obstante, en aquel momento ni las cartas ni el estudio crítico fueron publicados. El tono que la correspondencia había tomado hizo que tanto Schutz como Parsons decidieran conservarla como un asunto privado. El intercambio "culminó en una frustración mutua y, del lado de Schutz, con la garantía de que se abstendría de publicar el ensayo completo sobre el libro de Parsons EAS" (Wagner 1983:76). A pesar de esto, para fines del año 1969, los editores consiguieron los permisos tanto de la esposa de Schutz, Ilse, como de Parsons. En 1978, Richard Grathoff publica la correspondencia entre Parsons y Schutz bajo el título The Theory of Social Action: The Correspondence of Alfred Schutz and Talcott Parsons (en adelante TSA). El volumen incluye diez cartas, el artículo de Schutz y una visión retrospectiva del debate escrito por Parsons.

\subsection{El estudio crítico a EAS: consensos en torno a su lectura}

El estudio crítico de Schutz a EAS señala de modo enfático el excesivo énfasis puesto por Parsons en el estudio del ámbito de la teoría y en la "evolución" de los sistemas teóricos. En ese texto, el interés de Parsons se centra en el análisis de la teoría científica y en su evolución hacia una orientación normativa que recupera el punto de vista subjetivo. Esta es una de las características centrales que se dan entre 1920 y mediados de los sesenta y que, a nuestro entender, caracterizan el proyecto parsoniano, esto es, la idea de que el estudio de las ciencias es centralmente el estudio de las teorías científicas.

A juicio de Schutz, Parsons está en lo correcto cuando sostiene que una teoría de la acción carecería de sentido sin la aplicación del punto de vista subjetivo. De modo que existe un acuerdo entre Schutz y Parsons en torno a la relevancia del punto de vista subjetivo para la reflexión en ciencias sociales. En esta dirección, el acuerdo científico es posible sobre la base de que hay una serie de concordancias, la mayoría de las veces tácitas, que están en la base de las teorías científicas. Y "esos acuerdos no son estrictamente científicos o empíricos, sino que son acuerdos filosóficos y metodológicos, por lo menos, acerca, por ejemplo, de qué tipo de entidades estamos dispuestos a aceptar en el mundo, límite no solamente dado en términos empíricos, límite dado por lo que muchos llaman una cosmovisión" (Schuster 2002:52).

No obstante, según Schutz, Parsons "no sigue este principio hasta sus raíces" (TSA:36). Schutz realiza un análisis crítico de algunos temas de discusión que, a su entender, resultan incompatibles con el punto de vista subjetivo. Entre ellos puede mencionarse la distinción entre conocimiento de sentido común y conocimiento científico, el concepto de valores normativos, el concepto de acto unidad, así como cada uno de sus rasgos más salientes, los que presentan para Schutz la dificultad de sustituir el punto de vista subjetivo. La crítica schutziana hacía especial hincapié en dos elementos centrales: por un lado, el peligro que conlleva la sustitución de la realidad social por las abstracciones creadas por la ciencia; y, por el otro, el señalamiento de la necesidad de comprender esa realidad como resultado de la actividad humana. 
Es con relación a estos aspectos que Schutz articula su estudio crítico, resaltando principalmente la necesidad de recuperación del punto de vista subjetivo. Según Schutz, Parsons formula la pregunta: ¿qué significa este mundo para mí, el observador? Este tipo de ciencia social no trata directamente con el mundo de la vida cotidiana, común a todos nosotros, sino con "idealizaciones y formalizaciones" del mundo social, cuidadosamente seleccionadas: "esta posición no solo es posible sino que es aceptada por la mayoría de los científicos sociales". Esos científicos desarrollan y aplican "un refinado sistema de abstracciones acordes a este propósito que intencionalmente elimina al actor en el mundo social, con todos sus puntos de vista subjetivos" (TSA: 47). De esta forma, no tratan "directa e inmediatamente con el mundo de la vida, común a todos nosotros, sino con idealizaciones y formalizaciones del mundo social seleccionadas de modo hábil y conveniente". Sin embargo, esa pregunta debe ser reemplazada por otra: "Qué significa el mundo social para el actor observado dentro de ese mundo y qué quiso significar con su acción dentro de él". Esta referencia al punto de vista subjetivo puede y debe siempre realizarse (TSA: 47-48). Siempre es posible, y necesario, retroceder (go back) al "hombre olvidado" (forgotten man) de las ciencias sociales.

Los comentarios de Schutz refieren al problema del objetivismo en la producción de conocimiento y plantean la importancia de clarificar el sentido subjetivo, de modo de evitar el "olvido" y la sustitución de la realidad social. Esa reflexión, a juicio de Schutz, solo puede llevarse a cabo a partir de la comprensión filosófica. En ese marco, las cartas muestran el interés de Schutz de fundamentar filosóficamente, o en sus propias palabras, "ensanchar", "profundizar" y "complementar" el marco de referencia parsoniano y así recuperar ese fundamento de sentido "olvidado".

Esta intención se hace patente en algunos extractos de la correspondencia: "Usted me imputa de principio a fin, por tanto, una actitud antagónica hacia su posición, actitud que yo no he tenido en lo más mínimo. Por cierto, hay críticas a alguna de sus teorías en el artículo, y nunca he dudado en esclarecer los puntos en los cuales discrepo con usted. Pero me parece que la mayor parte de mi artículo muestra dónde y hasta qué punto nuestras teorías se unen" (TSA: 95).

También en este otro: “[Con relación a EAS] lo estudié detenidamente (...) e inmediatamente me di cuenta de la importancia y el valor de su sistema y también del hecho de que comienza exactamente donde mi propio libro termina (...) Encontré nuevos e interesantes puntos de convergencia y de divergencia con respecto a mi propio pensamiento" (TSA: 97, nuestro énfasis).

En un texto del año 1944 (Conceptos fundamentales de la fenomenología), puede encontrarse una frase similar: "buscando un verdadero comienzo de todo el pensamiento filosófico, la fenomenología espera terminar una vez plenamente desarrollada, allí donde comienzan las filosofías tradicionales. Su lugar está más allá -mejor dicho, antes- de todas las distinciones entre realismo e idealismo" (Schutz 2003:112). En ese marco, Schutz propone una articulación o "complementariedad" de los niveles de análisis, es decir, entre las categorías parsonianas -su marco de referencia de la acción- y un fundamento ontológico en el mundo de la vida -centrado en la reflexión filosófica en torno al tiempo y a la intersubjetividad-; 
articulación que permite restituir ese mundo olvidado y sustituido por las idealidades y las abstracciones del esquema parsoniano.

A pesar de esa intención de complementariedad, los intérpretes y comentaristas de la correspondencia entendieron cosas bastante diferentes. La interpretación posterior del debate constituye una buena muestra del modo en el que la teoría social ha sostenido una interpretación dominante de la fenomenología a través de lo que Michael Lynch denomina como "lecturas comunitarias" (Lynch 1993:13) erróneas de sus textos centrales. Esas lecturas comunitarias hacen referencia a un consenso virtual constituido por profundos malentendidos que han sido sostenidos a través de un conjunto común de eslóganes.

Esto nos llevaría a la discusión del fenómeno de cómo puede ser sostenida la comunicación académica a través de la creación sistemática de zonas de equívocos mutuos. Sin embargo, no nos interesa profundizar en este tema aquí, nos interesa en cambio señalar el modo en el que esas lecturas comunitarias introducen el dualismo, en primer lugar, en la obra de Schutz y, en segundo lugar, en el análisis de la correspondencia entre éste y Parsons.

Desde ese marco hermenéutico, se interpretan las contribuciones de ambos autores como proyectos antitéticos y contrapuestos. Esa "antítesis" se fundamenta en un análisis de la obra de Weber y en la posibilidad de escisión de su perspectiva teórica. Los intérpretes hacen una lectura dualista de la obra de Schutz, al suponer que éste escinde aquello que en Weber aparece vinculado: el significado subjetivo respecto del objetivo. En este sentido se sostiene que "Schutz radicaliza la perspectiva subjetiva, por el contrario, Parsons pone de relieve fundamentalmente la perspectiva objetiva" (Schwinn 1993:12). Y del mismo modo, fruto de una "ambivalencia meta-teórica de la sociología weberiana", en las perspectivas de Schutz y Parsons "se pone en juego una 'doble direccionalidad' del pensamiento social" (Endress 2009:385). Desde este punto de vista, "Schutz es calificado de subjetivista y Parsons de objetivista" (López 2012:50).

El mismo Parsons había llegado a esta conclusión respecto del trabajo de Schutz en su estudio retrospectivo: "El punto de vista fenomenológico, particularmente en la versión representada por Schutz, me parece un intento relativamente modesto de dar especial preponderancia a la parte subjetiva (...) del dilema cartesiano o post-cartesiano. Sin embargo, como he sugerido, al hacer esto se ha inclinado demasiado en la dirección de atribuir un estatus cuasi-metafísico al conocimiento inmediato de lo subjetivo y la correspondiente disminución de la importancia atribuida a ciertos roles especiales de la objetivación" (Parsons 1974:118).

A continuación presentaremos los argumentos más relevantes, lo que nos permitirá avanzar en dirección a la recuperación del proyecto epistemológico del fenomenólogo austríaco. 
López, D. 2014. El problema del objetivismo en la producción de conocimiento sociológico: La correspondencia entre Alfred Schutz, Talcott Parsons y Harold Garfinkel

\subsection{El proyecto epistemológico de Schutz: la fundamentación fenomenológica de las ciencias sociales}

Como he demostrado en trabajos anteriores (López 2012), intérpretes reconocidos de la obra de Schutz han problematizado los mencionados consensos en torno a la lectura errónea de las orientaciones del autor. Para Wagner, desde la perspectiva de los extensos escritos metodológicos de Schutz, el reproche acerca de que otorga más importancia a la parte subjetiva no coincide con su posición real. Para Schutz, el asunto fundamental es el de la relación entre el substrato originario de las experiencias humanas y los intentos de tratarlo (come to terms with) cognitiva y teóricamente. En este sentido, Schutz presta atención de igual manera a ambos lados de la cuestión.

Retrospectivamente, "Parsons polariza claramente la problemática de modo que no hace justicia a su complejidad" (Wagner 1980:388). Como sostiene Srubar, "las diferencias entre ambos enfoques no pueden ser vistas a la luz de la contraposición entre los puntos de partida subjetivo u objetivo" (Srubar 1988:201). En esta misma línea, Rehorick afirma que mientras Parsons impuso un esquema conceptual en el mundo social, Schutz hace de tales "imposiciones" su tema de indagación. Comenzando a construir desde el polo subjetivo, Schutz buscó trazar un puente entre las categorías subjetivas y objetivas: "[d]e ahí que Schutz buscara los orígenes de las categorías propias de las ciencias sociales en los hechos más fundamentales de la vida de la conciencia" (Rehorick 1980:354).

El propósito de trazar un puente entre las categorías subjetivas y objetivas que permitiese superar el problema del objetivismo en la producción de conocimiento en ciencias sociales, fue un objetivo que Schutz se planteó de modo muy claro ya en su obra temprana. Un examen detallado de las discusiones que motivaron su obra de juventud permite demostrar que el problema del objetivismo y la superación del dualismo fue el tópico central que movilizó su proyecto teórico y epistemológico.

Esas discusiones son las de la Escuela Austríaca de Economía, círculo intelectual cuya influencia sobre el pensamiento Schutz es ampliamente reconocida. La tendencia de la Escuela Austríaca hacia el objetivismo, el cual "dicotomiza en extremo (over-dichotomizes) teoría e historia y conocimiento científico del conocimiento de la vida cotidiana" (Lavoie 1994:57-58), es analizada como una limitación por los propios miembros de la Escuela. La misma surge de la mano de las reflexiones de su iniciador, Carl Menger, y "será heredada por la tercera generación de la Escuela centrada en Ludwig von Mises" (López 2014b:10).

Schutz se incorpora al seminario Mises (Miseskreise) en el año 1920 con el objeto de investigar y discutir la metodología de Weber y su potencialidad para el análisis económico. Mises y sus estudiantes aceptaron los principios fundamentales de Menger. Una muestra de la herencia recibida por Mises es su concepto de acción, el cual también dicotomiza teoría e historia dado su carácter a priori y su desvinculación respecto de las condiciones históricas y empíricas: "[a]unque los hombres de la Edad Media no hubiesen comprendido la ley de la utilidad marginal, ellos no podrían haber actuado de un modo distinto al descrito por la ley de la utilidad marginal" (Mises citado en Barber 2004:53). 
La teoría acerca de los rasgos a priori de la acción y la preferencia detrás de todas las acciones, las cuales pertenecen a todas las épocas y culturas, es denominada por Mises "praxeología". De acuerdo a Scarano, "[l]a praxeología (...) es teórica y sistemática, es una ciencia no histórica. Su alcance es la acción humana como tal, sin importar las circunstancias individuales de los actos. Es puramente formal y general. Sus enunciados no se derivan de la experiencia. Son como los enunciados de la lógica y la matemática, $a$ priori, no están sujetos a verificación ni falsificación en base a la experiencia (...) La cognición praxeológica es conceptual, es cognición de “universales y categorías'” (Scarano 2004:9).

A pesar de insistir en que la praxeología debe enfocarse en las percepciones subjetivas, las preferencias, los propósitos, las valuaciones y las expectativas de los actores, muchos economistas austríacos consideran a la praxeología como una ciencia anterior a toda experiencia que provee verdades universalmente válidas (Storr 2010:156). Este rasgo que caracteriza las construcciones científicas lo lleva a Mises a establecer una línea divisoria tajante entre teoría e historia y entre las verdades objetivas y las percepciones subjetivas de los actores. Es importante resaltar que Schutz parte del problema del objetivismo, del mismo modo que Mises y sus colegas en el marco del seminario.

Fundamentalmente la formulación de la pregunta: “¿Cómo son posibles las ciencias de contexto subjetivo de significado?" (Schutz 1972:251), es lo que organiza el último capítulo de su obra de juventud. Schutz recupera el interrogante central de los economistas austríacos de su generación, impregnando su primera obra de continuas referencias a la mencionada problemática. A su juicio es necesario radicalizar la revolución subjetivista, si lo que se pretende es evitar el peligro que tal perspectiva conlleva.

En este marco, CSM constituyó un intento por resolver el problema del objetivismo que la Escuela arrastraba desde hacía décadas. En ese libro, Schutz señala que el problema de la epistemología y la metodología de las ciencias sociales radica en la confusión acerca del estatuto del mundo social y enfatiza que: "[e]s cuestión de urgente necesidad que la filosofía de las ciencias sociales esclarezca las complejas relaciones existentes entre las diferentes dimensiones del mundo social, las someta a un análisis radical como para llegar a sus fundamentos mismos, y fije los límites entre sus diferentes estratos. Pues la controversia acerca del objeto propio de la metodología de las ciencias sociales es precisamente el resultado de la confusión acerca de estas cuestiones" (Schutz 1972:40).

En esa búsqueda de un análisis radical, el tiempo y la intersubjetividad constituyen el núcleo de la fundamentación fenomenológica de la sociología comprensiva y la clave para la superación de la crisis epistemológica. Solo después de clarificar estas cuestiones fundamentales, consideraba Schutz que se podía encarar el problema de la formación de conceptos en ciencias sociales. En este marco, sostiene que la estructura significativa del mundo tiene sus raíces, en primer lugar, en el nivel vivencial del yo solitario y, en segundo lugar, en los niveles de la vivencia social -tanto directa como indirecta. 
Desde el primer punto de vista, el mundo de la vida se presenta como una estructura de configuraciones de significado, las cuales se ordenan de forma gradual, desde los estratos de significado inferiores, más cercanos a la durée, hasta las configuraciones superiores de significado constituidas, ya no penetradas por el rayo de atención, lo que le permite a Schutz dar cuenta de la transición del polo subjetivo al polo objetivo. Por otra parte, y en relación con el mundo de los contemporáneos, la noción de "tipo" será central en el problema de la formación de conceptos y en la epistemología de las ciencias sociales: el contemporáneo sólo es indirectamente accesible y sus vivencias solo pueden conocerse en forma de tipos generales de vivencia.

El hecho de que el mundo de los contemporáneos y el de los predecesores puedan únicamente aprehenderse de una manera típico ideal, sirve de fundamento para los tipos ideales de los científicos sociales: "[p]uesto que es experiencia tipificante, la ciencia social constituye un contexto objetivo de significado, cuyo objeto, sin embargo, consiste en contextos subjetivos de significado (para ser más precisos, los procesos subjetivos típicos de los tipos personales ideales)" (Schutz 1972:251).

Schutz se ocupa claramente de distinguir los tipos ideales empleados por el actor en la vida cotidiana y los tipos ideales construidos por el científico social. La sociología comprensiva debe construir tipos personales ideales para actores sociales, que sean compatibles con los construidos por estos últimos. Este debe ser su postulado básico si se quiere evitar la separación de la teoría de su contacto con el mundo de la vida. Del mismo modo, los tipos ideales pueden ir de la excesiva concreción al anonimato total a partir de los procesos de formalización y generalización. La descripción puede ser empírica o eidética y el grado de anonimato de los constructos ideales (utilizados en cada ciencia social) determinará su actitud respecto del contexto subjetivo de significado de que ella trata. El resultado es una jerarquía de tipos ideales teóricos de anonimidad creciente. Las diferentes disciplinas pueden ubicarse a lo largo de ese continuo y, en este sentido, ciencia social teórica e histórica se reconcilian.

En suma, el razonamiento en términos dualistas constituye un sesgo de la teoría sociológica contemporánea que diluye el problema que está en el centro de la discusión: el problema epistemológico del objetivismo en la producción del conocimiento sociológico y el consecuente "olvido" del mundo de la vida por parte de los científicos sociales.

Para Schutz el problema del objetivismo en la producción del conocimiento consiste en la aceptación ingenua por parte de los científicos sociales del mundo social con todos los alter ego y las instituciones como un universo significativo, es decir, significativo para el observador, cuya única tarea consiste en "describir y explicar lo que el mundo significa para los observadores científicos", abandonando de este modo la explicación y la descripción de lo que significa para los actores dentro de su mundo social.

Esa intención no solo se hace clara en CSM, sino que se extiende a los intercambios con Parsons en los que Schutz plantea la complementariedad de su propuesta respecto del marco de referencia de la acción estructural funcionalista. Esta afirmación también se encuentra presente en los intercambios que Schutz mantuvo con Garfinkel. Allí no solo es posible encontrar una interpretación retrospectiva de Schutz sobre su correspondencia con Parsons a la luz del problema del objetivismo en la producción de 
conocimiento en ciencias sociales, sino que también revela que esa problemática ocupaba un lugar central en la reflexión de quien más tarde sería el fundador de la etnometodología. Sin embargo, como mostraremos, la respuesta de Garfinkel no será filosófica sino eminentemente metodológica.

\section{La correspondencia Schutz-Garfinkel}

Garfinkel se introdujo en la fenomenología como estudiante en la Universidad de Carolina del Norte entre los años 1939 y 1942. En algunos cursos de filosofía en los que participó se presentaban textos de Edmund Husserl, Marvin Farber y Schutz. En el año 1946 comienza sus estudios en Harvard y es en ese momento en el que Parsons se convierte (junto con Frederick Mosteller y Robert Freed Bales) en tutor de su tesis de doctorado. El mismo año conoce a Aaron Gurwitsch con quien discute temas vinculados a la fenomenología y a la sociología. Gurwitsch le recomienda a Garfinkel que le escriba a Schutz. Garfinkel estaba interesado en el asesoramiento y la opinión de Schutz acerca de su trabajo, particularmente, porque en éste retomaba muchas de sus contribuciones.

En el año 1949 Garfinkel le escribe a Schutz informándole que se encuentra completando su tesis de doctorado bajo la dirección de Parsons y solicitándole una reunión por recomendación de Gurwitsch. Garfinkel se encontraba, en aquel momento, trabajando en el problema del orden social bajo la dirección de Parsons, no obstante, consideraba necesario profundizar su análisis en dirección a una reflexión fenomenológica.

De ese modo, comienza una correspondencia y una serie de encuentros cara a cara con Schutz. Garfinkel viaja regularmente para "pasar tardes de tutoría" en New York (citado en Psathas 2009:402). En lugar de plantear una crítica directa a Parsons -su director-, Garfinkel analiza de modo comparativo la obra de su tutor y la de Schutz, lo que le permite seguir una perspectiva alternativa. Resulta interesante que, a pesar de que Parsons fue su director de tesis, "la misma representa una evaluación crítica y un rechazo a favor de Schutz" (Psathas 2009:411). De este modo, la profundización en la obra de Schutz le permitió a Garfinkel realizar una evaluación crítica de la perspectiva parsoniana.

En una carta del 8 de Octubre de 1953, Garfinkel le envía a Schutz dos trabajos de su autoría atravesados de modo notorio por las reflexiones epistemológicas de Schutz. El primero titulado "Cuatro decisiones pre-teóricas de Talcott Parsons y Alfred Schutz", el segundo, "Notas sobre la actitud sociológica". Estos textos inéditos, escasamente tematizados en el ámbito de la reflexión sociológica, permiten no solo recuperar los análisis retrospectivos de Schutz respecto de sus intercambios con Parsons, sino también rastrear la preocupación epistemológica de Garfinkel por el problema del objetivismo en la producción de conocimiento en ciencias sociales.

\subsection{La interpretación garfinkeliana de los vínculos Schutz-Parsons}

En el primer escrito, Garfinkel detalla cuatro problemas epistemológicos a partir de los cuales pueden compararse las decisiones de Schutz y Parsons, aclarando que su intención no es la de criticar esas decisiones, sino la de establecer un criterio comparativo con el fin de observar el modo en que las 
diferencias en las decisiones metodológicas y epistemológicas "derivan (eventuate) en diferentes concepciones sostenidas por los dos hombres con relación al problema del orden social" (Garfinkel 1953a:1).

En ese trabajo, el autor menciona una serie de problemas epistemológicos que enfrentan tanto Schutz como Parsons y analiza las distintas decisiones tomadas por ambos para dar respuesta a cada uno de ellos. Esos problemas se refieren, en primer lugar, a la necesidad de desarrollar una teoría de los objetos; en segundo lugar, a la determinación del estatus lógico y los usos de los tipos ideales empíricos; en tercer lugar, a la reflexión en torno a la posición del observador como parte del campo de observación; y, por último, al análisis del estatus lógico y los usos de las categorías "subjetivas".

Con relación al primer problema, Garfinkel sostiene que toda teoría de los objetos debe hacer referencia a cuestiones epistemológicas pues, a fin de definir tal teoría, se vuelve necesario utilizar términos tales como "objeto real" y "conocimiento del mundo objetivo". Tal decisión es tomada por Parsons a favor de una "teoría de la correspondencia" (correspondence theory) de la realidad. Mientras que la decisión de Schutz puede ser denominada "teoría de la congruencia" (congruence theory) de la realidad (Garfinkel aclara que retoma ambos términos de Felix Kaufman).

La teoría de la correspondencia de la realidad sostiene que al teorizar acerca del mundo se parte del supuesto de que existe una diferencia entre el objeto del mundo externo "percibido" y el "objeto concreto". De esto se sigue que nunca puede agotarse el número de afirmaciones fácticas que pueden hacerse acerca de un objeto. La teoría de la correspondencia sostiene, además, que la función de cualquier esquema categorial consiste en aportar cierto tipo de aproximación al objeto concreto. De tal manera, un modelo analítico debe juzgarse no solo con referencia a su utilidad en el marco de los propósitos del observador, sino también en relación con la medida en que éste aporta una reproducción precisa de la realidad que está "ahí afuera".

Desde esta teoría, tal precisión es un resultado automático si se actúa de acuerdo a un correcto respeto de los cánones de la indagación lógico-empírica. Además, la teoría de la correspondencia realiza una separación entre el mundo real y la interpretación subjetiva del mundo real. La separación es tal que no hay, por un lado, objetos reales en toda su completitud y, por el otro lado, representaciones conceptuales de esos objetos. Desde esta perspectiva, tales representaciones "al abstraer ciertos rasgos de los objetos concretos lo enfrentan al científico con una reproducción degradada" (Garfinkel 1953a:4).

Por otra parte, la premisa destacada de la "teoría de la congruencia" de la realidad sostiene que el objeto del "mundo exterior" percibido es un objeto concreto y que los dos términos "objeto percibido" y "objeto concreto" son sinónimos y términos intercambiables. Esta visión plantea, en contraste con la teoría de la correspondencia, que las evidencias sensoriales son las condiciones pero no los contenidos de la percepción. La concreción solo es posible desde el momento en que hay un estándar fundamentado en el orden no-perceptual el cual permanece invariante bajo los diferentes aspectos sensoriales. La teoría de la congruencia no es más que una paráfrasis de la correlación entre noesis y noema, la que Garfinkel denomina como "la relación entre objeto y actitud” (Hama 2009:445). 
Un objeto real es cualquier conjunto de experiencias unificadas sin tomar en consideración el hecho de que éstas se fundamenten de modo ideal o sensorial. De esta forma, la plenitud del objeto concreto no proviene del énfasis esquemático en la representación conceptual del objeto. Por el contrario, el objeto concebido nunca aparece excepto a través de su esquema. El esquema de especificaciones es precisamente el objeto mismo. El carácter concreto del objeto se encuentra en el objeto constituido como una unidad de significado. De tal manera, habrá realidades múltiples, una pluralidad de mundos de significado cada uno constituido por su particular actitud: "Mientras que en la teoría de la correspondencia puede formularse la pregunta acerca de qué es el mundo objetivo y qué es el conocimiento objetivo, solamente es posible preguntarse bajo las premisas de la teoría de la congruencia, cuáles son los distintos mundos objetivos y cuáles son las variantes del conocimiento objetivo" (Garfinkel 1953a:6).

En segundo lugar, con relación al estatus lógico y al uso de los tipos ideales, Parsons parte del hecho de que el lenguaje no es constitutivo del objeto. Asimismo, asume que las representaciones abstractas sirven como estándares arbitrarios de comparación con los acontecimientos reales y que el objeto es independiente de ellas. Por su parte, para Schutz, el objeto es constituido de acuerdo a los propósitos del individuo y, por lo tanto, la cuestión de la relevancia se transforma en algo importante si lo que se intenta es describir los rasgos estructurales del objeto. En este sentido, el tiempo y el espacio del actor así como su sistema de relevancias son aspectos de la organización de la unidad de significado y esa organización no es una aproximación al objeto sino el objeto tal como se le aparece al actor. El lenguaje es, por tanto, inseparable de la unidad de significado. El tipo ideal es, entonces, el objeto construido por el actor en tanto orientado hacia éste.

En tercer lugar, con relación al tópico del observador como parte de su campo de observación, puede sostenerse que, en la teoría de la correspondencia, el observador es quien decide si el conocimiento del mundo que posee el actor es exacto y quien provee, metodológicamente hablando, el criterio para decidir qué es para el actor una cuestión de creencia, fantasía, hecho, teoría o sentimiento. El hombre racional y el ideal racional del científico comparten "la comunidad de un mundo considerado racionalmente" (Psathas 2009:408). Cada uno de ellos puede encontrar la verdad si sigue los procedimientos racionales.

Para Schutz tales nociones son suspendidas. No se parte ni de la noción de comunidad ni de la de hombre racional, sino que la pregunta se concentra en tratar de comprender el modo en que las personas "construyen, mantienen, alteran, validan, cuestionan y definen un orden juntos" (Garfinkel 1953a:19). El actor y el observador "actúan solo sobre la base de aquello que saben" y la respuesta a sus acciones debe buscarse en las construcciones y en la lógica de la vida cotidiana, en la estructura de relevancias y en lo que es adecuado para el propósito a mano.

Por último, con relación al estatus lógico de las categorías subjetivas, en el marco de la teoría de la correspondencia parsoniana se establece una diferencia entre la percepción del objeto y el objeto concreto. En la separación entre sujeto y objeto, la única función de las construcciones empíricas es que 
López, D. 2014. El problema del objetivismo en la producción de conocimiento sociológico: La correspondencia entre Alfred Schutz, Talcott Parsons y Harold Garfinkel

resultan útiles en términos de "economía" de actividad. La separación entre sujeto y objeto requiere que Parsons inserte "entre" ellos la noción de relación: "El sujeto permanece relacionado al sistema de objetos a través de sus orientaciones de acción" (Garfinkel 1953a:21). La definición de la situación por parte del actor es considerada por Parsons como una variable interviniente. Por el contrario, para Schutz la interpretación subjetiva es el postulado básico del método utilizado por el sociólogo en cada momento de su actividad de investigación. De hecho, Schutz desaprueba el uso del término "categorías subjetivas" por considerarlo engañoso puesto que, al ser utilizado frecuentemente por Parsons para establecer una línea divisoria entre el mundo representado por las categorías objetivas y el mundo representado de acuerdo a las categorías subjetivas, se muestra a esos "dos mundos" como diferentes uno del otro.

Con estas comparaciones se abre el camino para demostrar el modo en que estas decisiones conducen a diferentes concepciones del problema del orden: "las diferentes decisiones los conducen a diferentes expresiones de la pregunta y, de este modo, difieren las visiones con relación a la solución correcta" (Garfinkel 1953a:23). Según Garfinkel, del mismo modo en que "el observador parsoniano se sitúa, en términos epistemológicos, externamente respecto de su objeto de indagación, la definición del problema del orden social se establece a partir de un criterio 'externo' al mundo social, ese criterio es el del propio observador" (López 2014a:103).

La carta que oficia como respuesta al manuscrito de Garfinkel presenta elementos que apoyan lo expresado más arriba, esto es, la insistencia de Schutz en torno a su afinidad y complementariedad con el pensamiento de quien, desde la interpretación dominante, es su oponente. Así comienza la respuesta de Schutz a Garfinkel: "Al leer sus interpretaciones de la teoría de Parsons y la mía, no estoy tan seguro como usted asume- de que haya realmente tales diferencias fundamentales entre nuestras 'decisiones' (...) ¿Podría, en su lugar, ser interpretada la diferencia entre Parsons y yo como una diferencia en el nivel de investigación? Parsons piensa que la investigación empírica, si es llevada adelante lo suficientemente lejos y se agrupa de acuerdo a un esquema conceptual, conducirá necesariamente a interpretaciones (insights) en torno a problemas, los que solo pueden ser tratados en un nivel puramente teórico. Yo, al comenzar por una filosofía básica, trato de explicar los hechos empíricos como aplicaciones especiales de las interpretaciones (insights) obtenidas a partir del análisis fenomenológico de las estructuras de la conciencia" (Schutz 1954:1, nuestro énfasis).

La consecuencia de esto, a juicio de Schutz, es que Parsons cae en contradicciones puesto que espera que al coordinar los hallazgos de la sociología, la psicología conductista, la antropología, el psicoanálisis y los resultados de la investigación de los grupos pequeños, podrá realmente alcanzar una teoría de la acción con ciertas categorías básicas, tales como roles, expectativas, etc. Sin embargo, para Schutz resulta que todas esas categorías básicas que son el resultado de su investigación son equívocas y ambiguas, "conté al menos siete significados del término expectativa tal como es utilizado por él". Y lo mismo es válido para los otros conceptos fundamentales en su teoría: "Por mi parte, trato de comenzar con la aplicación del análisis de Husserl de la estructura de la vida consciente al problema del mundo social, lo que para mí es, básicamente, el mundo de la intersubjetividad tal como es experimentado en la actitud natural. A partir de aquí, trato de explicar y de elucidar el fenómeno concreto del mundo social 
definiendo el tema de las ciencias sociales como el intento de explicar ese fenómeno en términos de las experiencias que poseen en su actitud natural las personas que viven su vida cotidiana dentro él, y en términos del significado que esas experiencias poseen para ellos. En una palabra, mi ideal sería terminar donde Parsons comienza" (Schutz 1954: 2, nuestro énfasis).

\subsection{Garfinkel y la actitud sociológica: la imposibilidad fundamental}

El segundo texto que Garfinkel le envía a Schutz comienza con un análisis de las diferencias entre la actitud natural y la actitud sociológica. Según Garfinkel, el sociólogo se gana la vida tratando las cosas familiares como extrañas. Su empresa de "mirar con ojos de sociólogo" lo involucra en un proceso de transformación de aquello que es familiar y conocido en la vida diaria en algo altamente problemático y para nada obvio. Sin embargo, el señalamiento de las ironías provocadas por la empresa resultante de este modo de tratar al mundo por parte de los sociólogos guiados por estos preceptos es solo una parte del asunto.

Garfinkel está interesado en mostrar la existencia de propiedades de la actitud que rige la tarea de "mirar sociológicamente" (Garfinkel 1953b:1). Para ello, a fin de describir la actitud científica sociológica, el autor se propone proveer un conjunto de reglas de procedimiento que expliquen el modo en el que se adopta esa actitud. Fundamentalmente, desde la perspectiva del joven Garfinkel el problema del objetivismo en la producción de conocimiento deriva de las discrepancias entre el "ideal de la actividad científica que los sociólogos consideran como el estándar al que buscan aproximarse en sus propias actividades" (Garfinkel 1953b:2), esto es, las reglas "ideales" de la actividad, y las reglas con las que "realmente" éstos trabajan.

Esencialmente, la respuesta al problema del objetivismo en la producción del conocimiento consiste en el reconocimiento de la existencia de esas discrepancias y en la descripción de las medidas que se emplean para encararlas. De este modo, Garfinkel describe a la ciencia en términos de las "actividades de las personas reales, las actividades reales que están regidas -en un grado que puede ser investigablepor las reglas que se instrumentan para lograr, en alguna medida, una aproximación al ideal de la actividad científica" (Garfinkel 1953b:3). En términos generales, para Garfinkel, el científico social busca aproximarse al ideal de poner al mundo entero en duda, ideal que conlleva la búsqueda de una desvinculación respecto de ese mundo.

La propuesta de Garfinkel consiste en evaluar las actividades de los sociólogos tomando en consideración el grado en el cual sus actividades y las reglas que de hecho las rigen se aproximan a tal ideal. El análisis realizado desde una perspectiva "ideal", o lo que Garfinkel denomina la actitudcientífica-en-general, no presenta diferencias con la teorización en las ciencias naturales. Desde otro punto de vista, al referirse a las características específicas de la actitud sociológica, Garfinkel sostiene que éstas pueden encontrarse en los postulados (en "el vocabulario metodológico" que puede ser denominado como "reglas básicas de procedimiento") que rigen el modo en el que los sociólogos construyen y manipulan los distintos esquemas de interpretación -es decir, sus teorías-, y que utilizan en el curso del ordenamiento de los datos que refieren a la conducta humana. 
A diferencia de lo que se piensa habitualmente, la actitud teórica en sociología no requiere que el teórico emplee un vocabulario sistemático de términos sociológicos, en el sentido, por ejemplo, de la teoría "estructural-funcionalista" de Parsons. De hecho, puede teorizarse sociológicamente con vocabularios que no solo son asistemáticos, sino que son recogidos de campos cuyas "jergas interpretativas" suenan desconocidas para los sociólogos. Lo cierto es que es imposible decir qué teoría debe emplearse a fin de que se la considere como un correcto "mirar sociológicamente". Las cuestiones que delimitan la mirada sociológica no tienen que ver con la teoría particular que se esté utilizando, sino con las reglas en el marco de las cuales se procede, tanto para la construcción como para el uso de esa teoría. Puesto que "estas reglas son las que en efecto regirán el uso y, de esta manera, determinarán los significados de los términos de un vocabulario particular" (Garfinkel 1953b:21).

En esta dirección, Garfinkel se ocupa de definir las reglas operacionales de la actitud sociológica. En principio, existen dos reglas que sirven para definir a la perspectiva sociológica en términos "operacionales". Esas reglas refieren a las condiciones operacionales que hacen posible que se considere que una persona -cuyas observaciones de cualquier evento de conducta humana y cuyo uso de un vocabulario que nombra e interpreta aquello que presencia (witnesses), observa e interpreta en el marco de esas reglas- está observando y teorizando sociológicamente.

Esas reglas son: la "regla del empirismo sociológico" y la "regla del marco de la acción". Con respecto a la primera, Garfinkel sostiene que desde el momento en que tomamos como nuestro objeto de investigación aquello que las personas dicen y hacen, debemos -con relación a nuestro tratamiento del contenido total de su actividad-, por un lado, abstenernos de emitir cualquier juicio en lo referente a esa actividad y, por el otro, toda nuestra descripción y teorización debe realizarse de modo tal de respetar los límites impuestos por esa abstención.

De acuerdo a Garfinkel, un acto de juicio (act of judgment) refiere al "carácter real" de los objetos, esto es, a su carácter "ontológico". Esta regla comporta un procedimiento doble que permite a los críticos justificar los fundamentos que se utilizan para rechazar la adecuación científica de una descripción o interpretación de ciertos eventos de conducta. En primer lugar, con relación a la descripción, el observador puede realizarla de forma tal que el objeto descrito pueda reproducirse, en su totalidad, en la experiencia de cualquier otro observador que establezca las condiciones provistas por las afirmaciones descriptivas, como condiciones bajo las cuales esa experiencia puede ser reproducida. En lo que respecta a cuestiones de hecho, esta regla prevalece como el único fundamento científicamente legítimo para establecer un consenso con respecto al reconocimiento o al rechazo de un cuerpo de conocimiento científico de cualquier afirmación que pretenda explicar cómo es el mundo.

En segundo lugar, con relación a la interpretación, el observador debe construir su esquema interpretativo de modo tal que, tomado en sí mismo, éste posea meramente un "estatus analítico", es decir, que no contenga conceptos ni afirmaciones que dependan para su significado de la suscripción por parte del observador a una teoría de la existencia. El esquema interpretativo debe estar construido de modo tal de presentar solo un conjunto de reglas que permitan relacionar afirmaciones de hecho, esto 
es, de modo tal de no realizar afirmaciones de hecho en sí mismas. Es decir, a la teoría solo se le está permitido decir qué significa el mundo y, en este sentido, solo puede referirse al modo en el que las afirmaciones fácticas deben relacionarse una con otra. Sin embargo, no se le permite decir en qué consiste el mundo de hecho. La regla del empirismo sociológico descarta de cuajo toda reflexión de carácter ontológico. Y este punto es importante pues implica un distanciamiento de Garfinkel respecto de la reflexión ontológica.

Por otra parte, la segunda regla se refiere al marco de la acción. Esta regla no significa que el observador tenga que ponerse en el lugar de la otra persona sino que refiere al modo en el cual se le permite al observador construir el mundo del actor una vez construido su tipo ideal, su "títere". Del mismo modo que es un sinsentido decir que el físico puede ponerse en el lugar de un átomo típico y ver las cosas como éste las ve, es un sinsentido decir que el científico social puede hacer esto con sus personas típicas.

Garfinkel se propone evaluar las actividades de los sociólogos con referencia al grado en el cual sus actividades y las reglas que las rigen de hecho se aproximan al ideal científico. De acuerdo al autor, debemos preguntarnos, en primer lugar, acerca de los puntos de discrepancia, y en segundo, por las decisiones metodológicas sobre las cuales se basan las prácticas reales en comparación con las decisiones metodológicas que deberían haberse tomado a fin de hacer coincidir la práctica real con lo que requiere el ideal; esto es, la fuente de discrepancias.

Para Garfinkel, el estudio de las decisiones metodológicas sobre las cuales se basan las prácticas reales de los sociólogos es prácticamente un territorio desconocido. A este tipo de conocimiento Garfinkel refiere como "tierra incógnita". Existen muchas fuentes de discrepancia, por ejemplo, el problema del observador como parte del campo de observación y el ideal que requiere una mirada desde una perspectiva impersonal, entre otros. Lo que es importante de destacar es que la imposibilidad de que el ideal de la actitud científica sociológica pueda ser experiencialmente satisfecho permanece como una alternativa abierta y abre el espacio de investigación sobre el conjunto de reglas operacionales que guía la actividad de "mirar sociológicamente". El reconocimiento de esa imposibilidad permite dar respuesta al problema del objetivismo en la producción del conocimiento, es decir, el reconocimiento de la imposibilidad de un ajuste automático del ideal científico y de las teorías sociológicas respecto de su objeto abre un nuevo horizonte hermenéutico que se propone como tarea describir las reglas operacionales que articulan el punto de vista sociológico.

\section{Consideraciones finales}

Tal como fue mencionado, el problema epistemológico del objetivismo en la producción de conocimiento sociológico confronta al investigador con la pregunta acerca del riesgo que trae consigo la sustitución de la realidad social por las idealidades y las abstracciones creadas por la ciencia. La tematización de ese tópico es una tarea ineludible que debe abordar la reflexión en ciencias sociales de cara a una adecuada fundamentación de sus conceptos científicos. En el artículo se recuperaron, en primer lugar, los aportes de la fenomenología de Schutz desde un análisis de la correspondencia que éste mantuvo con Parsons. 
Como se mostró, el autor discute ampliamente las dificultades por las que atraviesan las ciencias sociales con relación a la fundamentación epistemológica de sus conceptos y propone una respuesta filosófica que consiste en la articulación de una ontología del mundo de la vida que recupera ese fundamento de sentido "olvidado" que es la realidad social. En esta misma dirección, mostramos en segundo lugar, el interés de Garfinkel por ese debate epistemológico entre Schutz y Parsons. Mediante la recuperación de sus textos de juventud inéditos pudimos, por un lado, fundamentar nuestro argumento en torno a la intención de complementariedad del proyecto schutziano con el estructural funcionalismo parsoniano, de modo de dar respuesta al problema del objetivismo en la producción de conocimiento. Por otra parte, revelaron que ese mismo problema ocupaba un lugar central en la obra temprana de Garfinkel, permitiendo establecer una continuidad con los problemas debatidos en la correspondencia epistolar entre Schutz y Parsons.

Sin embargo, desde una perspectiva hermenéutica, y en oposición a la "historia del problema" (Gadamer) de corte neokantiano, no resulta posible plantear una identidad en la formulación del problema por estos autores, sino que es necesario examinar las interrogaciones y la conceptualidad propia de cada uno de ellos. En base a estas consideraciones, en el proyecto epistemológico que se plantea en la obra temprana de Garfinkel, recuperamos otra formulación y otra respuesta al problema del objetivismo en la producción de conocimiento en sociología.

El problema del objetivismo en la producción de conocimiento en sociología parte de una imposibilidad fundamental: la concreción en términos experienciales del ideal de la actitud científica sociológica. El reconocimiento de esa imposibilidad abre el espacio de investigación acerca del conjunto de reglas operacionales que guía la actitud sociológica. Esto debe realizarse, en primer lugar, identificando los puntos o fuentes de discrepancia, y en segundo, investigando las decisiones metodológicas sobre las cuales se basan las prácticas reales, en comparación con las decisiones metodológicas que deberían haberse tomado a fin de hacer coincidir la práctica real con lo que requiere el ideal.

En suma, en base a la recuperación de sus textos de juventud inéditos, pudimos afirmar que el autor también parte del problema del objetivismo en la producción de conocimiento. Sin embargo, a diferencia de Schutz, niega la posibilidad de una perspectiva ontológica y articula una respuesta de carácter metodológico. El autor propone un abordaje que pone el foco en la "mirada" desde la que la sociología describe la acción social, de modo de iluminar los razonamientos prácticos y las decisiones metodológicas de los investigadores, a fin de evitar la sustitución de la realidad social por las idealidades de la reflexión científica.

Si bien Garfinkel, desde principios de los años 60, se aparta de la sociología con su propuesta etnometodológica, pues diferencia explícitamente su etnometodología respecto del abordaje de la sociología y se refiere a ambos abordajes como "inconmensurables" (la etnometodología se plantea como diferente de la sociología, puesto que es necesariamente una ciencia empírica que no requiere, como lo hace la sociología convencional, del nivel de lo hipotético, de lo típico o de las instancias idealizadas), decíamos, es posible sostener que, en el momento de la correspondencia entre Garfinkel y 
López, D. 2014. El problema del objetivismo en la producción de conocimiento sociológico:

Schutz, todavía existe en Garfinkel un anclaje en la reflexión sociológica: "Puede sostenerse que, en este momento, Garfinkel continúa bajo la influencia del pensamiento de Alfred Schutz, no obstante, al mismo tiempo, se encuentra trabajando en lo que más adelante será su propio programa [la etnometodología]" (Psathas 2004:18), y es a esa reflexión hacia donde apuntó el presente trabajo.

En efecto, la lectura de la obra temprana de Garfinkel pone al descubierto su preocupación en torno al problema del objetivismo en la producción de conocimiento sociológico, específicamente en la descripción científica de la acción social: "lo que hace el observador tradicional, de acuerdo a Garfinkel, es partir de la perspectiva de la reflexión científica, la cual reifica al actor" (Rawls 2006:16). Es por ese motivo, que el autor plantea la necesidad de determinar las propiedades de la actitud que rigen la tarea de "mirar sociológicamente" y que se ponen en juego en la práctica sociológica.

Garfinkel se propone arrojar luz sobre los razonamientos prácticos y las decisiones metodológicas de los investigadores en sociología, a fin de evitar la sustitución del mundo social por las idealidades científicas y plantear un desarrollo del conocimiento científico no escindido de las realidades concretas de los actores sociales y su vida cotidiana. En sus propias palabras se propone: "describir la actitud científica sociológica; proveer un grupo de reglas procedimentales que establecen el modo en que debe adoptarse la actitud sociológica; y mencionar algunos problemas particulares a las tareas propias de aquellos que llevan adelante sus investigaciones de acuerdo a esas reglas" (Garfinkel 1953b:1).

En base a estos textos sostuvimos que, a diferencia de Schutz, la respuesta que articulará Garfinkel no será filosófica sino eminentemente metodológica: “la metodología se refiere a la teoría de las decisiones científicas correctas y no al estudio de la teoría sociológica misma o al estudio de las técnicas de investigación (...) [y] el estudio de las decisiones metodológicas sobre las cuales están basadas las prácticas reales de los sociólogos es prácticamente un territorio desconocido (...) una 'terra incognita'” (Garfinkel 1953b:28).

La respuesta de Schutz a ese programa fue de estímulo: "si usted piensa que el estudio de las decisiones metodológicas sobre las cuales están basadas las prácticas reales de los sociólogos son un territorio prácticamente desconocido, tiene mi total aprobación y espero que sea usted uno de los exploradores de esa isla del tesoro aún no explorada" (Schutz 1954:3, énfasis en el original). Garfinkel fue incentivado por Schutz a iluminar esos problemas y a profundizar su indagación. En este sentido, entendemos que el tratamiento de esas cuestiones permite esclarecer las derivaciones metodológicas de la reflexión schutziana en la obra temprana de Garfinkel. Siendo la respuesta de Schutz esencialmente teórica, Garfinkel argumenta que esa actitud teórica es responsable de muchos problemas en la investigación social, sentando una posición crítica respecto de tal planteo del problema: "[e]l pensador teórico, al permanecer en la actitud teórica, no puede experimentar y alcanzar con inmediatez el mundo de la vida cotidiana" (Garfinkel 2006:128).

Por su parte, la posición garfinkeliana sostiene que el conocimiento de las prácticas no consiste en un conocimiento conceptual o cognitivo, sino en un conocimiento "hecho cuerpo" (embodied) que surge de la participación en esas prácticas y en la co-presencia de Otros: "[c]on la atención suficiente en las 
prácticas en detalle es posible aprender a 'mirar' que es lo que se ha estado haciendo, mirar de un nuevo modo, mirar los detalles de las prácticas situadas, en lugar de realizar reducciones conceptuales, esto es, la práctica de 'mirar sociológicamente'"' (Rawls 2006:91).

En suma, la recuperación de esos acervos bibliográficos inéditos de la obra temprana de Garfinkel, los cuales han sido escasamente estudiados en el ámbito de la discusión especializada, permite rastrear en ellos una epistemología de inspiración fenomenológica que ofrece un abordaje empírico distintivo para investigar la producción de conocimiento en sociología.

\section{Agradecimiento}

Deseo agradecer al Servicio Alemán de Intercambio Académico (DAAD) por hacer posible una estancia de investigación en el Archivo de las Ciencias Sociales (Archivo en Memoria de Alfred Schutz) de la Universidad de Konstanz. Así como a su director, el Dr. Jochen Dreher, por facilitar el acceso a los materiales inéditos de Garfinkel.

\section{Nota}

Todas las citas del inglés y del alemán son de mi traducción.

\section{Bibliografía}

Barber, M. 2004. The participating citizen. A biography of Alfred Schutz. Albany: State University of New York Press.

Endress, M. 2009. Two directions of continuing the Weberian proyect: Alfred Schutz and Talcott Parsons. En: H. Nasu, L. Embree, G. Psathas y I. Srubar (Eds.) Alfred Schutz and his intellectual Partners. Konstanz: UVK Verlagsgesellschaft mbH, pp. 377-400.

Garfinkel, H. 1953a. A comparison of decisions made of four 'pre-theoretical' problems by Talcott Parsons and Alfred Schutz. En: Schütz's Papers, Alfred Schütz Gedächtnis Archiv, Sozialwissenschaftliches Archiv, Universität Konstanz, Alemania.

Garfinkel, H. 1953b. Notes on the sociological attitude. Schütz's Papers, Alfred Schütz Gedächtnis Archiv, Sozialwissenschaftliches Archiv, Universität Konstanz, Alemania.

Garfinkel, H. 2006. Seeing sociologically. The routine grounds of social action. Boulder/London: Paradigm Publishers.

Hama, H. 2009. The Primal Scene of Ethnomethodology: Garfinkel's Short Story 'Color Trouble' and the Schutz-Parsons Controversy. En: H. Nasu, L. Embree, G. Psathas y I. Srubar (Eds.), Alfred Schutz and his Intellectual Partners. Konstanz: UVK Verlagsgesellschaft mbH, pp. 435-449.

Lavoie, D. 1994. The interpretive turn. En: P. Boettke (Ed.) The Elgar companion to Austrian economics. Northampton: Edward Elgar, pp. 54-62. 
López, D. 2014. El problema del objetivismo en la producción de conocimiento sociológico: La correspondencia entre Alfred Schutz, Talcott Parsons y Harold Garfinkel

Lynch M. 1993. Scientific practice and ordinary action. Ethnomethodology and social studies of science. Cambridge: Cambridge University Press.

López, D. G. 2012. The oblivion of the life-world. The correspondence of Alfred Schutz and Talcott Parsons. Schutzian Research IV: 47-64.

López, D. G. 2014a. El 'Schutz objetivista'. Aportes de las reflexiones schutzianas al problema del orden social. En: J. Dreher y D. López (Eds.) Fenomenología del poder. Bogotá: Universidad Santo Tomás, pp. 83-115.

López, D. G. 2014b. The epistemic claim to the life-world: Alfred Schutz and the debates of the Austrian school of economics. The Review of Austrian Economics doi 10.1007/s11138-014-0280-x

Parsons, T. 1974. A 1974 retrospective perspective. En: A. Schutz y T. Parsons. The theory of social action: the correspondence of Alfred Schutz and Talcott Parsons. Bloomington/London: Indiana University Press, pp. 115-124.

Psathas, G. 2004. Alfred Schutz's influence on American sociologists and sociology. Human Studies 27(1): 1-35. doi: 10.1023/B:HUMA.0000012249.47522.56

Psathas, G. 2009. The correspondence of Alfred Schutz and Harold Garfinkel: what was the "terra incognita" and the "treasure island"? En: H. Nasu, L. Embree, G. Psathas y I. Srubar (Eds.). Alfred Schutz and his intellectual partners. Konstanz: UVK Verlagsgesellschaft mbH, pp. 401-433.

Rawls, A. W. 2006. Respecifying the study of social order - Garfinkel's transition from theoretical conceptualization to practices in details. En: H. Garfinkel. Seeing sociologically. The routine grounds of social action. Boulder/London: Paradigm Publishers, pp. 1-99.

Rehorick, D. 1980. Schutz and Parsons: Debate or dialogue? Human Studies 3(4): 347-355.

Scarano, E. R. 2004. El apriorismo de Ludwig von Mises. Revista Libertas 40

http://www.eseade.edu.ar/servicios/Libertas/1 2 Scarano.pdf

Schuster, F. 2002. Filosofía y métodos de las ciencias sociales. Buenos Aires: Manantial.

Schutz, A. 1954. Letter to Harold Garfinkel on January 19, 1954. Schütz's Papers, Alfred Schütz Gedächtnis Archiv, Sozialwissenschaftliches Archiv, Universität Konstanz, Alemania.

Schutz, A. 1972. La construcción significativa del mundo social. Buenos Aires: Paidós.

Schutz, A. 2003. El problema de la realidad social. Escritos I. Buenos Aires/Madrid: Amorrortu Editores.

Schwinn, T. 1993. Jenseits von Subjektivismus und Objektivismus: Max Weber, Alfred Schütz und Talcott Parsons. Berlin: Duncker \& Humblot. 
Storr, V.H. 2010. Schütz on objectivity and spontaneous orders. Schutzian Research 2: 165-181.

Srubar, I. 1988. Kosmion: Die Genese der pragmatischen Lebenswelttheorie von Alfred Schütz und ihr anthropologischer Hintergrund. Frankfurt a. M.: Suhrkamp.

Wagner, H. R. 1980. Reflections on Parsons' "1974 retrospective perspective" on Alfred Schutz. Human Studies 3(4): 387-402.

Wagner, H. R. 1983. Alfred Schutz: an intellectual biography. Chicago and London: The University of Chicago Press.

Recibido el 24 ene 2014

Aceptado el 16 jun 2014 